08

\title{
Модификация и полировка штриха голографической дифракционной решетки пучком нейтрализованных ионов $\mathrm{Ar}$
}

\author{
(C) С.А. Гарахин, М.В. Зорина, С.Ю. Зуев, М.С. Михаленко, А.Е. Пестов, Р.А. Плешков, \\ В.Н. Полковников, Н.Н. Салащенко, Н.И. Чхало
}

Институт фозиики микроструктур РАН, 607680 Нижний Новгород, Россия

e-mail: GarakhinS@yandex.ru

Поступило в Редакцию 13 апреля 2020 г.

В окончательной редакции 13 апреля 2020 г.

Принято к публикации 13 апреля 2020 г.

\begin{abstract}
Описан метод увеличения эффективности голографических дифракционных решеток (ГДР), предназначенных для рентгеновского диапазона длин волн. Описана процедура обработки поверхности ГДР пучком ускоренных ионов с целью понижения амплитуды и шероховатости штрихов. Для понижения амплитуды штриха применялись ионы Хе с энергией $600 \mathrm{eV}$, для ионной полировки - ионы $\mathrm{Ar}$ с энергией $800 \mathrm{eV}$. Показано увеличение дифракционной эффективности решетки на длине волны $4.47 \mathrm{~nm}$ почти в 4 раза после ионной полировки.
\end{abstract}

Ключевые слова: дифракционная решетка, монохроматор Черни-Тернера, ЭУФ диапазон длин волн, ионная полировка поверхности, атомно-силовая микроскопия.

DOI: 10.21883/JTF.2020.11.49976.130-20

\section{Введение}

Для совершенствования технологии изготовления оптических элементов для экстремального ультрафиолетового и мягкого рентгеновского диапазонов необходимы данные контроля рентгенооптических свойств (спектральные, угловые, поляризационные зависимости коэффициентов отражения/пропускания, индикатрисы рассеяния и др.) синтезированных образцов на рабочих длинах волн. Ориентация только на синхротроны сильно ограничивает возможности исследователей. Практика показала, что для создания высококачественных многослойных структур необходима оперативная информация об их свойствах, с целью проведения анализа и оптимизации процесса синтеза без разрыва технологической цепочки, поэтому тенденцией последних лет стало создание многофункциональных лабораторных рефлектометров [1-6].

Для этих целей на базе спектрометра-монохроматора со схемой Черни-Тернера с двумя коллимирующими зеркалами и плоской дифракционной решеткой [7] авторами был разработан рефлектометр, описанный в [8]. В качестве дифракционных решеток использовались голографические решетки производства Государственного института прикладной оптики, г. Казань, предназначенные для работы в диапазоне длин волн с коротковолновой границей $50 \mathrm{~nm}$ [9]. Поэтому эффективность использования голографических дифракционных решеток (ГДР) в диапазоне длин волн короче $50 \mathrm{~nm}$ требует дополнительных исследований.

В настоящей работе исследовались шероховатость и профиль штрихов, наиболее сильно влияющие на дифракционные свойства решеток в скользящей геометрии, используемой в мягком рентгеновском диапазоне.
Демонстрируется возможность существенного влияния на шероховатость и форму штрихов с помощью травления ионным пучком, что позволяет улучшить их рентгенооптические характеристики.

\section{1. Постановка задачи}

В настоящей работе исследовались две ГДР с геометрическими размерами $10 \times 60 \times 75 \mathrm{~mm}$ (рис. 1). Штрихи наносились параллельно высоте $60 \mathrm{~mm}$. Периоды решеток $D$ составляли 1.111 и $3.333 \mu \mathrm{m}$ или 900 и 300 lines/mm соответственно. Основные уравнения для

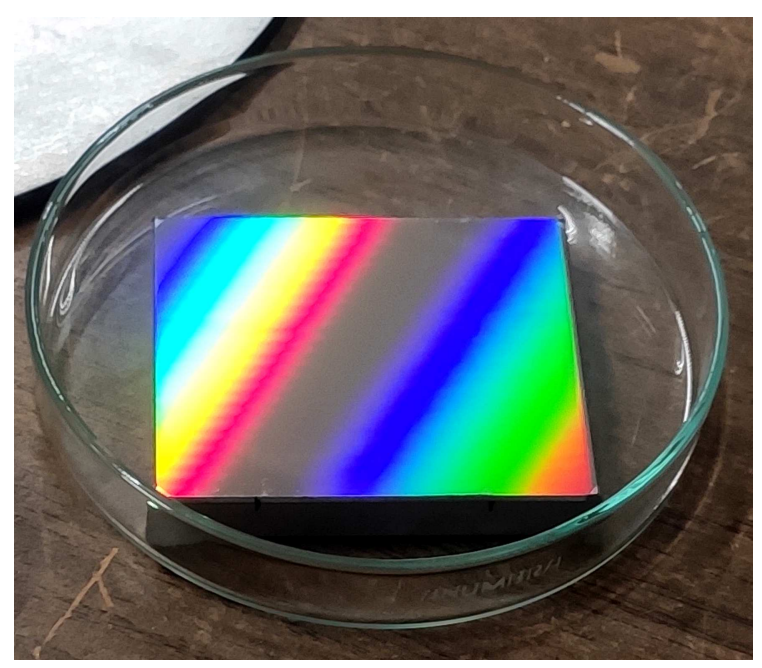

Рис. 1. Фотография ГДР с плотностью штрихов $300 \mathrm{~mm}^{-1}$ производства ГИПО, г. Казань. 

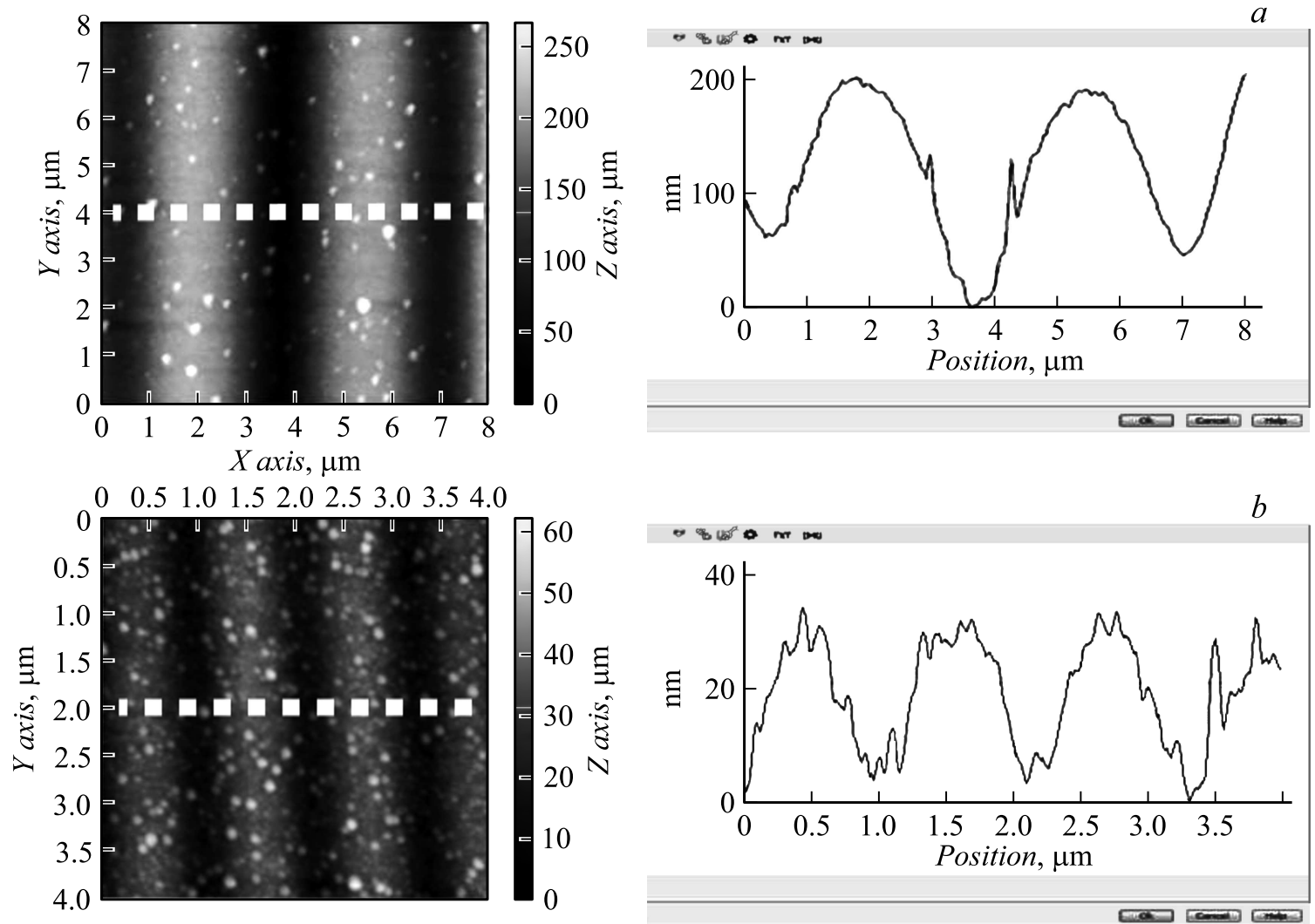

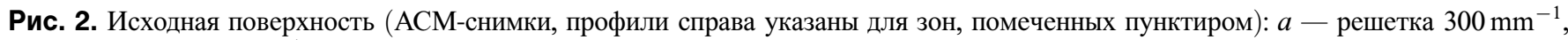
$b$ - решетка $900 \mathrm{~mm}^{-1}$.

схемы Черни-Тюрнера, устанавливающие связь между длиной волны $\lambda$, максимальной рабочей длиной волны $\lambda_{\max }$, углами падения и дифракции $\alpha$ и $\beta$, и периодом решетки следующие:

$$
\cos \alpha-\cos \beta=m \cdot \lambda / D,
$$

где $m$ - порядок дифракции, сумма углов падения и дифракции П остается постоянной и не зависит от длины волны

$$
\begin{gathered}
\Phi=\alpha+\beta, \\
\lambda_{\max }=D \cdot(1-\cos \Phi) .
\end{gathered}
$$

В нашем случае $\Phi=12^{\circ}$ (угол определяется конструкцией прибора), поэтому соотношение (3) можно переписать в виде

$$
\lambda_{\max }=2.185 \cdot 10^{-2} \cdot D .
$$

Соответственно первая решетка имеет длинноволновую границу рабочего диапазона $\lambda_{\max 1}=24 \mathrm{~nm}$, вторая $\lambda_{\max 2}=72 \mathrm{~nm}$.

Из соотношения (2) вытекает одна особенность применения этих решеток в скользящей геометрии. Для того чтобы решетка эффективно работала, сумма углов падения и наклона штриха решетки должна быть меньше величины $\Phi$. В противном случае штрих решетки будет экранировать дифрагированное в направлении выходной щели спектрометра-монохроматора излучение.

Другая очевидная особенность решеток для рентгеновского диапазона - это высокие требования к шероховатости поверхности штрихов, связанные с короткой длиной волны излучения. Поэтому по аналогии с [10], где ионной полировкой удалось существенно повысить эффективность нарезных дифракционных решеток, в настоящей работе решались следующие задачи. Первая - исследовать шероховатость и форму штрихов решеток. Для этих целей использовалась атомно-силовая микроскопия (ACM). Вторая - изучить возможность сглаживания поверхности и изменения высоты штрихов с помощью ионной полировки. Третья - изучить влияние ионной обработки на эффективность дифракционных решеток.

\section{2. Исследование характеристик профиля ГДР}

Изучение характеристик профиля ГДР проводилось на стенде ACM, позволяющем исследовать крупногабаритные детали [11]. На рис. 2 приведены АСМ кадры фрагментов поверхности решеток (слева) и сечения, перпендикулярные штрихам (справа), для решеток $300 \mathrm{~mm}^{-1}$ (рис. 2,a) и $900 \mathrm{~mm}^{-1}$ (рис. 2,b). Из при- 

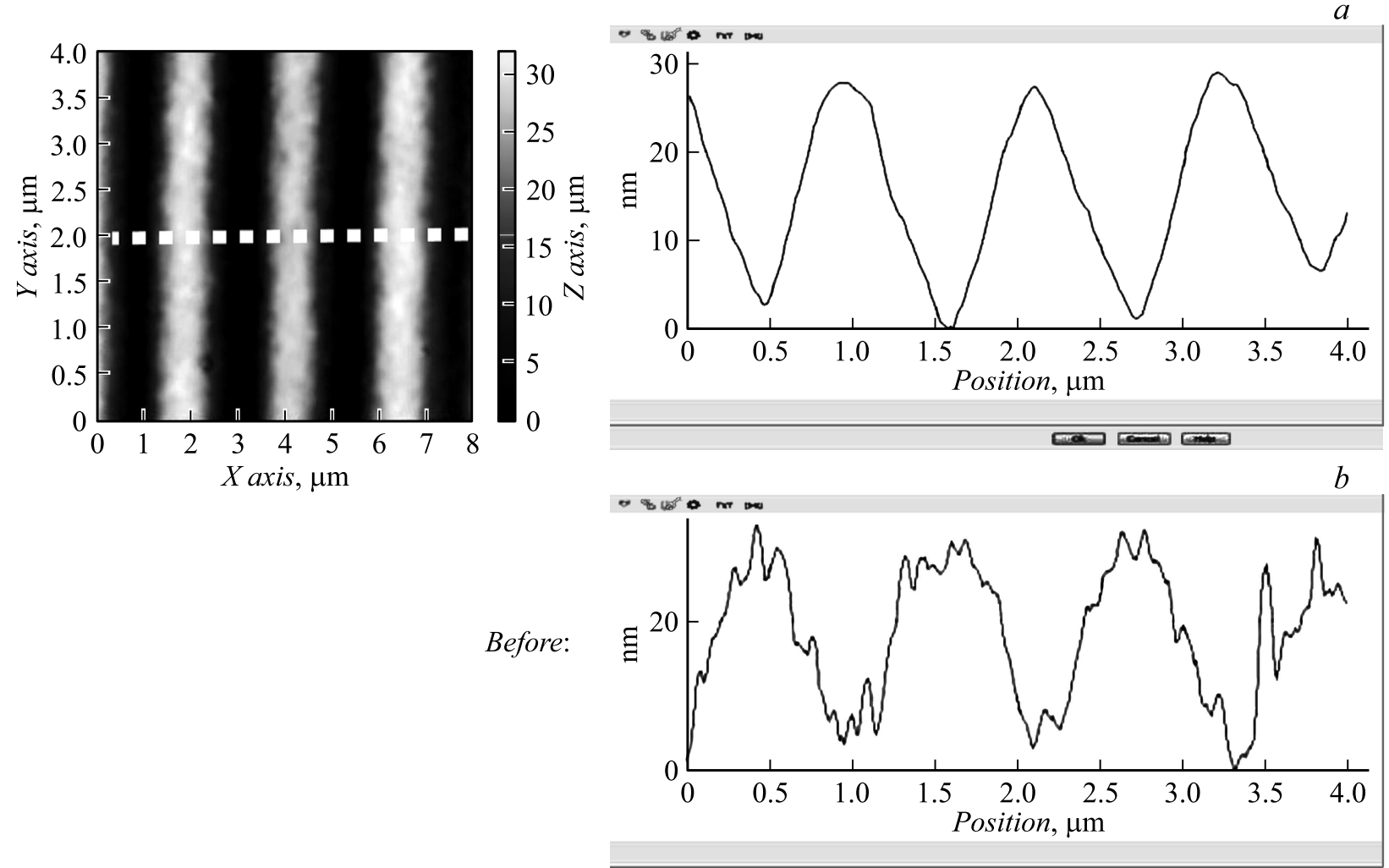

एक

Рис. 3. АСМ изображение и перпендикулярное сечение для ГДР $900 \mathrm{~mm}^{-1}$ после первого травления. Снизу приведено перпендикулярное сечение исходной решетки.

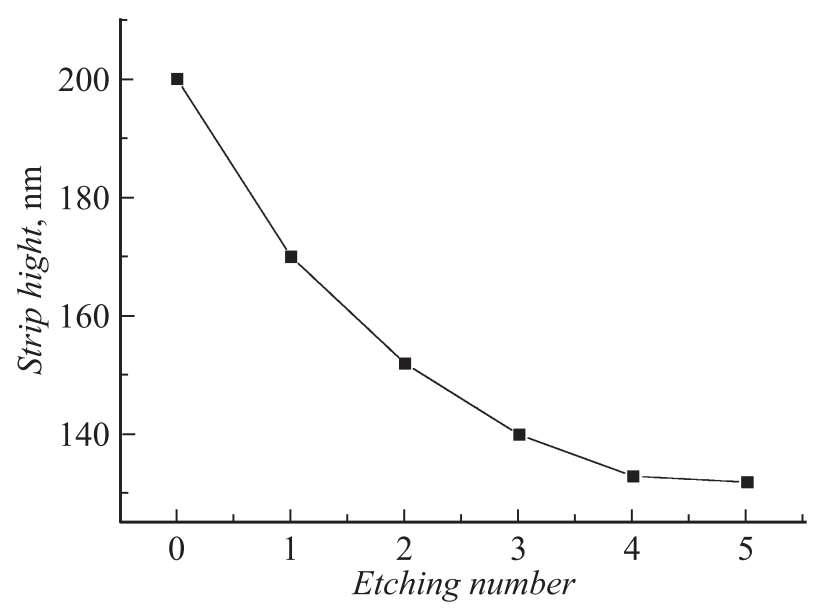

Рис. 4. Зависимость высоты штриха дифракционной решетки $\left(300 \mathrm{~mm}^{-1}\right)$ от количества операций травления.

веденных данных можно сделать следующие выводы. Первое, поверхность имеет большую шероховатость. Так как решетки покрывались пленкой алюминия, то наблюдаемые на поверхности пики (белые точки), повидимому, представляют собой кристаллиты алюминия. Второе, высоты штрихов у решетки $300 \mathrm{~mm}^{-1}$ имеют заметный разброс по величине, в то время как у ре- шетки $900 \mathrm{~mm}^{-1}$ разброс незначителен. Третье, высота штрихов $h_{g r}$ для решетки $300 \mathrm{~mm}^{-1}$ достигает $200 \mathrm{~nm}$, а для $900 \mathrm{~mm}^{-1}$ — около $30 \mathrm{~nm}$. Угол блеска $\gamma$ можно оценить из соотношения

$$
\gamma \approx 2 \cdot h_{g r} / D
$$

Если для второй решетки этот угол существенно меньше угла падения излучения на решетку, то для ГДР $300 \mathrm{~mm}^{-1}$ он достигает $6.9^{\circ}$. В сумме угол падения (дифракции) и наклона превышают $\Phi=12^{\circ}$. Это означает, что эффективность решетки в спектрометре будет крайне низкой, так как отражать будет только вершина решетки, где наклон минимальный.

Таким образом, исследование характеристик профиля ГДР показало, что в исходном виде решетки не могут быть использованы в приборе для рентгеновского диапазона. Решетка $300 \mathrm{~mm}^{-1}$ - из-за большой глубины штриха, $900 \mathrm{~mm}^{-1}$ - из-за большой шероховатости.

\section{3. Влияние ионной полировки на поверхность ГДР}

Ранее в ряде работ, например [12-14], была показана возможность сглаживания (полировки) высокочастотной шероховатости пучками ускоренных ионов аргона. Оптимальные энергии ионов лежат в диапазоне 800-1000 eV. 

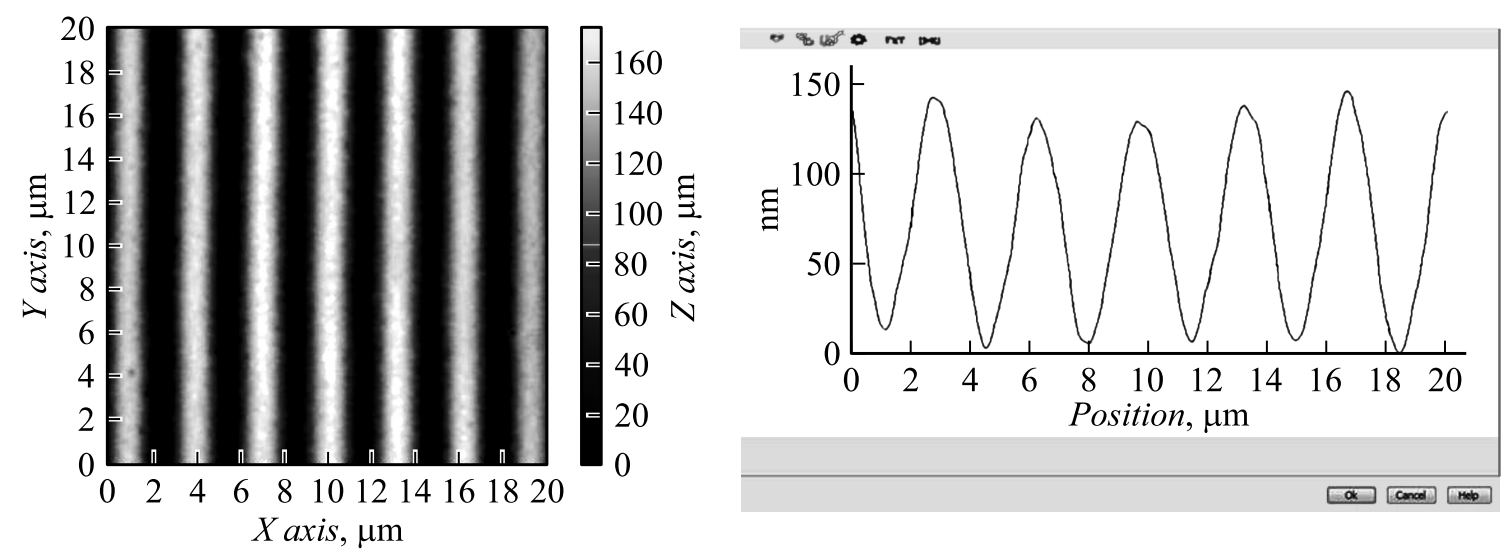

Рис. 5. АСМ изображение и перпендикулярное сечение для ГДР $300 \mathrm{~mm}^{-1}$ после пятого травления.

В [15] было показано, что ионы ксенона с оптимальной энергией $600 \mathrm{eV}$ позволяют расширить диапазон пространственных частот, для которых наблюдается сглаживание шероховатостей. Поэтому для решетки $300 \mathrm{~mm}^{-1}$, для которой требовалось и сглаживание шероховатости, и понижение профиля штриха (пространственная частота $0.3 \mu \mathrm{m}^{-1}$ ), использовались ионы ксенона. Для ГДР $900 \mathrm{~mm}^{-1}$ требовалось сглаживание высокочастотной шероховатости при сохранении глубины штриха (пространственная частота $0.9 \mu \mathrm{m}^{-1}$ ). Поэтому она обрабатывалась ионами аргона с энергией $800 \mathrm{eV}$.

Однако методика хорошо работает только для подложек из аморфных материалов и в редких случаях в очень узком диапазоне параметров процесса, для монокристаллических материалов [12,16]. Так как в нашем случае образцы были покрыты металлом, то мы использовали подход, предложенный в [17], хорошо зарекомендовавший себя при ионной полировке бериллиевых подложек. Суть подхода заключается в том, что на поверхность методом магнетронного распыления наносился $200 \mathrm{~nm}$ слой аморфного кремния, и ионная полировка уже велась по данному слою. Эксперименты проводились на установке, описанной в [18]. Модификации подвергалась вся площадь дифракционных решеток за исключением незначительных крепежных областей по углам.

Как правило, для сглаживания высокочастотной шероховатости достаточно одной итерации нанесение кремния - ионное травление. На рис. 3 приведены АСМ изображение и перпендикулярное сечение для ГДР $900 \mathrm{~mm}^{-1}$ после одной процедуры. Как видно из рисунка, если сравнить с исходной поверхностью (before), шероховатость упала сильно, а вот глубина профиля, как и ожидалось при травлении ионами аргона, практически не изменилась.

Для ГДР $300 \mathrm{~mm}^{-1}$ помимо полировки необходимо было уменьшить высоту штриха. Как оказалось, из-за достаточно низкой пространственной частоты $\left(0.3 \mu \mathrm{m}^{-1}\right)$ периода решетки потребовалось несколько итераций. На рис. 4 приведена зависимость амплитуды штриха от количества операций травления. Как видно, после 4-й процедуры нанесение кремния - ионное травление, высота штриха перестала изменяться, достигнув величины порядка $130 \mathrm{~nm}$, что соответствует углу наклона штриха около $4.5^{\circ}$. Этот угол уже позволяет использовать решетку в приборе.

АСМ изображение поверхности, а также его сечение поперек штрихов, приведены на рис. 5. Как можно видеть из рисунка, шероховатость практически исчезла, так же упал разброс высоты штрихов, что позволяет ожидать хорошей дифракционной эффективности и низкого уровня рассеянного на решетке излучения.

\section{4. Исследование абсолютной эффективности дифракции ГДР}

Исследования в экстремальном ультрафиолетовом и мягком рентгеновском диапазонах проводились на лабораторных рефлектометрах, описанных в [19] на характеристических линиях $\mathrm{C} K_{\alpha}(4.47 \mathrm{~nm}), \mathrm{B} K_{\alpha}(6.76 \mathrm{~nm})$, $\mathrm{YM}_{\xi} \quad(9.34 \mathrm{~nm}), \quad \operatorname{Be} K_{\alpha} \quad(11.4 \mathrm{~nm}), \quad \mathrm{Si} L_{\alpha} \quad(13.5 \mathrm{~nm})$, $\mathrm{Al} L_{\alpha}(17.14 \mathrm{~nm}), \quad \mathrm{Li}_{\alpha}(25 \mathrm{~nm}), \quad$ He II $(30.4 \mathrm{~nm})$ и Не I $(58.4 \mathrm{~nm})$. На рис. 6, a приведена угловая зависимость интенсивности дифрагированного излучения с длиной волны $4.47 \mathrm{~nm}$ от ГДР $900 \mathrm{~mm}^{-1}$ при фиксированном угле падения излучения на решетку $\theta_{\text {in }}=7^{\circ}$. Жирными символами обозначена кривая исходной решетки, мелкими - после ионной полировки. Как можно видеть, дифракционная эффективность выросла в 3 раза.

На рис. $6, b$ приведена зависимость эффективности в 1-м порядке ГДР $300 \mathrm{~mm}^{-1}$ от длины волны. Кривая снималась при фиксированном угле детектора по отношению к падающему излучению $\theta_{d}=12^{\circ}$, что моделировало условия в рефлектометре. Как видно из рисунка, после уменьшения высоты штриха ГДР $300 \mathrm{~mm}^{-1}$ может использоваться в составе спектрометра-монохроматора скользящего падения экстремального ультрафиолетового и мягкого рентгеновского излучения. 

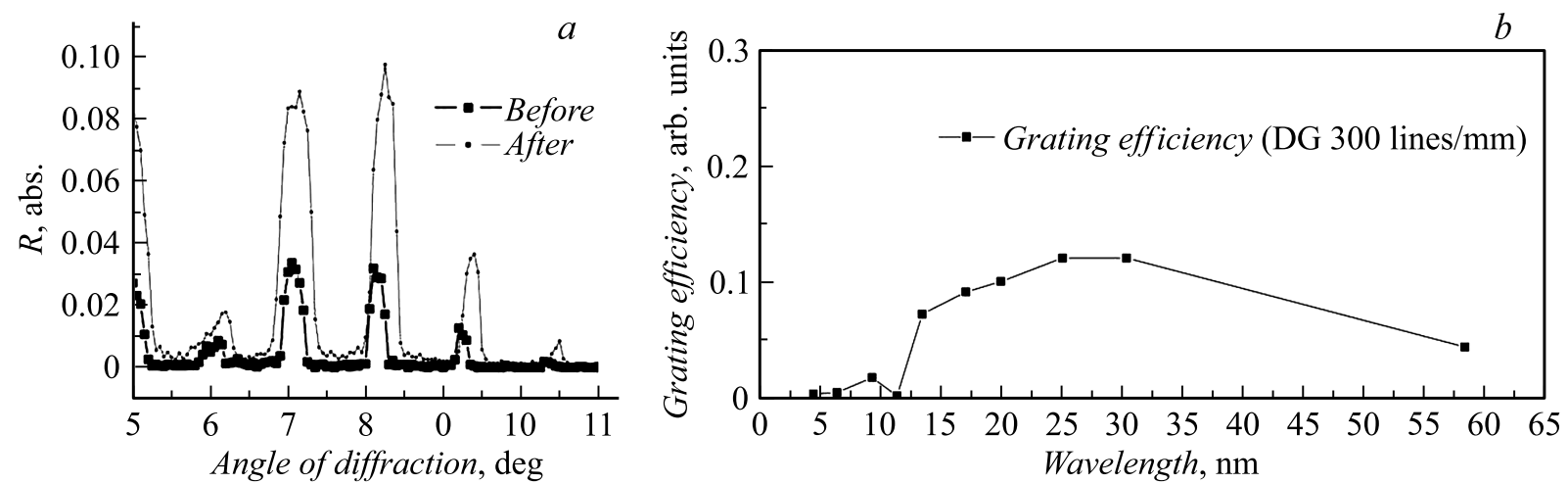

Рис. 6. $a$ - угловая зависимость интенсивности дифрагированного излучения с длиной волны $4.47 \mathrm{~nm} \mathrm{от} \mathrm{ГДР} 900 \mathrm{~mm}{ }^{-1}$ при фиксированном угле падения излучения на решетку $\theta_{\text {in }}=7^{\circ} ; b-$ зависимость эффективности в 1 -м порядке ГДР $300 \mathrm{~mm}^{-1}$ от длины волны, кривая снималась при фиксированном угле детектора по отношению к падающему излучению $\theta_{d}=12^{\text {circ }}$, что моделировало условия в рефлектометре.

\section{Заключение}

Целью работы было изучение возможности использования традиционных голографических решеток, предназначенных для работы в видимом и вакуумном ультрафиолетовом диапазонах, в экстремальном ультрафиолетовом и мягком рентгеновском диапазонах. Спецификой этого диапазона являются повышенные требования к шероховатости поверхности штрихов и скользящие углы падения, и дифракции излучения. В ходе исследования было показано, что в исходном виде, прежде всего из-за большой шероховатости, такие решетки имеют низкую эффективность и не могут использоваться в составе рентгеновских спектрометров-монохроматоров.

В работе описывается методика, позволяющая не только кардинально сгладить шероховатость, но и уменьшать высоту штриха. Суть методики заключается в ионно-пучковой обработке слоя аморфного кремния, предварительно нанесенного на обрабатываемую поверхность. Причем для полировки лучше использовать ионы аргона, а для понижения штриха - ионы ксенона. С использованием этого метода у ГДР $300 \mathrm{~mm}^{-1}$ высота штриха была уменьшена с исходных 200 до $130 \mathrm{~nm}$, что уменьшило минимальный угол отбора дифрагированного излучения с $6.9^{\circ}$ до $4.5^{\circ}$ и тем самым повысило эффективность дифракционной решетки в составе спектрометра-монохроматора скользящего падения для экстремального ультрафиолетового и мягкого рентгеновского излучения.

Полировка штрихов ГДР $900 \mathrm{~mm}^{-1}$ повысила дифракционную эффективность на длине волны $4.47 \mathrm{~nm}$ в 3 раза.

В заключении следует отметить, что описанный метод может представлять интерес не только для рентгенооптики, но и для традиционных диапазонов видимого и ультрафиолетового излучения. Ионно-пучковая обработка приведет к уменьшению светорассеяния от решетки, а также позволяет с высокой точностью подогнать высоту штриха, оптимальную для решения конкретных задач, что невозможно осуществить другими методами.

\section{Финансирование работы}

Работа выполнена в рамках выполнения государственного задания № 0035-2014-0204 и при поддержке грантов РФФИ №№ 20-02-00708, 19-32-90154, 18-02-00588 и 18-07-00633, с использованием оборудования ЦКП „Физика и технологии микро- и наноструктур“ при ИФМ PAH.

\section{Конфликт интересов}

Авторы заявляют, что у них нет конфликта интересов.

\section{Список литературы}

[1] Gullikson E.M., Underwood J.H., Batson P.C., Nikitin V. // J. of x-ray Sci. Technol. 1992. Vol. 3. N 4. P. 283.

[2] Loyen L., Boettger T., Braun S., Mai H., Leson A., Scholze F., Tuemmler J., Ulm G., Legall H., Nickles P., Sandner W., Stiel H., Rempel C., Schulze M., Brutscher J., Macco F., Muellender S. // Proc. SPIE. 2003. Vol. 5038. N 11.

[3] Miyake A., Miyachi T., Amemiya M., Hasegawa T., Ogushi N., Yamamoto T., Masaki F., Watanabe Y. Proc. SPIE 5037, Emerging Lithographic Technologies VII, 647. 2003.

[4] Wanga H., Wanga X., Chena B., Wanga Y., Maoa S., Rena S., Zhoua P., Liua Y., Huob T., Zhoub H. // Optik Intern. J. for Light and Electron Optics. 2020. Vol. 204. P. 164213.

[5] Scholze F., Bottger T., Enkisch H., Laubis C., Loyen L., Macco F., Schadlich S. // Meas. Sci. Technol. 2007. Vol. 18. P. $126-130$.

[6] Vishnyakov E.A., Kolesnikov A.O., Kuzin A.A., Negrov D.V., Ragozin E.N., Sasorov P.V., Shatokhin A.N. // Quant. Electron. 2017. Vol. 47. P. 1.

[7] Czerny M., Turner A.F. // Zeitschrift für Physik. 1930. Vol. 61. 
[8] Гарахин С.А., Забродин И.Г., Зуев С.Ю., Каськов И.А., Лопатин А.Я., Нечай А.Н., Полковников В.Н., Салащенко Н.Н., Цыбин Н.Н., Чхало Н.И. // Квант. электрон. 2017. T. 47. № 4.

[9] Электронный ресурс. Режим доступа: https://shvabe.com/upload/iblock/df0/DO-GDR-O.pdf

[10] Зорина М.В., Зуев С.Ю., Михайленко М.С., Пестов А.Е., Полковников В.Н., Салащенко Н.Н., Чхало Н.И. // Письма в ЖТФ. 2016. Т. 42. Вып. 16. С. 34-40.

[11] Chkhalo N.I., Salashchenko N.N., Zorina M.V. // Rev. Sci. Instrum. 2015. Vol. 86. P. 016102.

[12] Ziegler E. et al. // Nucl. Instrum. Methods Phys. Res. A. 2010. Vol. 616 (2-3). P. 188.

[13] Chen S. et al. // Appl. Opt. 2015. Vol. 54. N 6. P. 1478.

[14] Chkhalo N.I., Churin S.A., Mikhaylenko M.S., Pestov A.E., Polkovnikov V.N., Salashchenko N.N., Zorina M.V. // Appl. Opt. 2016. Vol. 55. P. 6.

[15] Chkhalo N.I., Churin S.A., Pestov A.E., Salashchenko N.N., Vainer Y.A., Zorina M.V. // Opt. Express. 2014. Vol. 22. P. 20094.

[16] Chkhalo N.I., Kirsanov A.V., Luchinin G.A., Malshakova O.A., Mikhailenko M.S., Pavlikov A.I., Pestov A.E., Zorina M.V. // Appl. Opt. 2018. Vol. 57. N 24. P. 6911-6915.

[17] Chkhalo N.I., Mikhailenko M.S., Mil'kov A.V., Pestov A.E., Polkovnikov V.N., Salashchenko N.N., Strulya I.L., Zorina M.V., Zuev S.Yu. // Surf. Coat. Technol. 2017. Vol. 311. P. 351-356.

[18] Chkhalo N.I., Kaskov I.A., Malyshev I.V., Mikhaylenko M.S., Pestov A.E., Polkovnikov V.N., Salashchenko N.N., Toropov M.N., Zabrodin I.G. // Precision Eng. 2017. Vol. 48.

[19] Bibishkin M.S., Chehonadskih D.P., Chkhalo N.I., Kluyenkov E.B., Pestov A.E., Salashchenko N.N., Shmaenok L.A., Zabrodin I.G., Zuev S.Yu. // Proc. SPIE. 2004. Vol. 5401. P. 8-15. 